\title{
Colon adenocarcinoma with dome-like phenotype: characteristic endoscopic ultrasonography (EUS) findings
}

Authors

Institutions
Wataru Takagi ${ }^{1}$, Katsumi Yamamoto ${ }^{1}$, Takahiro Amano ${ }^{1}$, Aisa Sakamoto ${ }^{1}$, Yuriko Otake ${ }^{1}$, Hirotsugu Saiki ${ }^{1}$, Hisashi Kondo ${ }^{1}$, Makiko Urabe ${ }^{1}$, Kei Takahashi ${ }^{1}$, Masashi Yamamoto ${ }^{1}$, Shiro Hayashi ${ }^{1}$, Sachiko Nakajima ${ }^{1}$, Tsutomu Nishida', Takamichi Komori², Shunji Morita ${ }^{2}$, Shiro Adachi ${ }^{3}$, Masami Inada ${ }^{1}$

Institutions are listed at the end of article. submitted

11. November 2014 accepted after revision 5. March 2015

\section{Bibliography}

DOI http://dx.doi.org/

10.1055/s-0034-1392019

Published online: 21.5.2015

Endosc Int Open 2015; 03:

E359-E362

(c) Georg Thieme Verlag KG

Stuttgart · New York

E-ISSN 2196-9736

\section{Corresponding author}

\section{Tsutomu Nishida, MD}

Department of

Gastroenterology

Toyonaka Municipal Hospital

4-14-1 Shibahara

Toyonaka

Osaka 560-8565

Japan

Fax: +81-6-68583531

tnishida.gastro@gmail.com
An 80-year-old man underwent colonoscopy for proctorrhagia. Conventional white-light imaging showed a superficially flat and elevated lesion that appeared to be a submucosal tumor of the sigmoid colon. Chromoendoscopy with Indigo Carmine showed that the margin of the tumor was covered with normal epithelium but that there was a slight depression on its surface. Magnification endoscopy with Crystal Violet staining revealed the amorphous surface structure of the depressed lesion, but the surrounding mucosa showed a normal pit pattern. Endoscopic ultraso-

\section{Introduction}

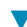

Adenocarcinoma of the colon with lymphoid stroma has been reported with two phenotypes: one is termed lymphoepithelioma-like carcinoma (LELC), and the other is termed dome-like carcinoma. LELC generally comprises undifferentiated carcinoma or medullary carcinoma with lymphoid stroma, which are typically associated with Epstein - Barr virus (EBV) infection and/or microsatellite instability-high colorectal cancer. LELC was originally described as a nasopharyngeal neoplasm in 1982 [1]. Since then, similar neoplasms have been described in several organs. In contrast, dome-like carcinoma of the colon was first reported in 2002 by Jass et al. as a distinct variant of colon cancer with a phenotype similar to that of LELC [2]. Unlike LELC, dome-like carcinoma is characterized by a well- and/or moderately differentiated histology with cystically dilated tumor glands, expansive growth, and confinement to an aggregate of lymphoid tissue [2], such as LELC. This tumor type is not associated with EBV infection and shows a microsatellite-stable phenotype. We herein report a case of colon adenocarcinoma with a dome-like phenotype of the sigmoid colon that was similar in appearance to a submucosal tumor (SMT) according to endoscopy. However, the presence of a demarcated area of depressed, irreg- nography demonstrated that a hypoechoic mass was located in the submucosal layer, and a biopsy specimen obtained from the surface of the lesion showed evidence of adenocarcinoma. We then performed sigmoidectomy on the patient. Immunohistochemically, the tumor cells were positive for two mismatch repair proteins (MLH1 and MSH2), but in situ hybridization revealed that the specimen was negative for the Epstein-Barr virus. We finally diagnosed the lesion as adenocarcinoma with a dome-like phenotype of the sigmoid colon.

ular mucosa on the tumor surface is a characteristic endoscopic ultrasonography (EUS) finding.

\section{Case report $\nabla$}

An 80-year-old man presented with proctorrhagia and underwent colonoscopy. He had no family history of colorectal cancer. Conventional white-light imaging revealed an elevated lesion of $10 \mathrm{~mm}$ in diameter with a flat top that appeared to be an SMT in the sigmoid colon ( $\bullet$ Fig. 1 ). Chromo-

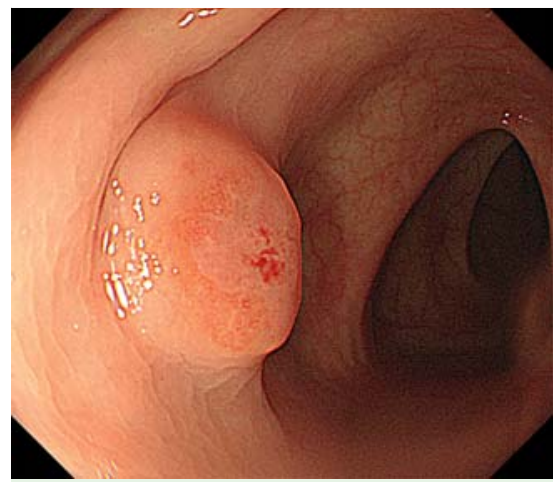

Fig. 1 White-light endoscopy image showing an elevated lesion with a flat top similar to a submucosal lesion in the sigmoid colon in an 80 year-old man who presented with proctorrhagia. 


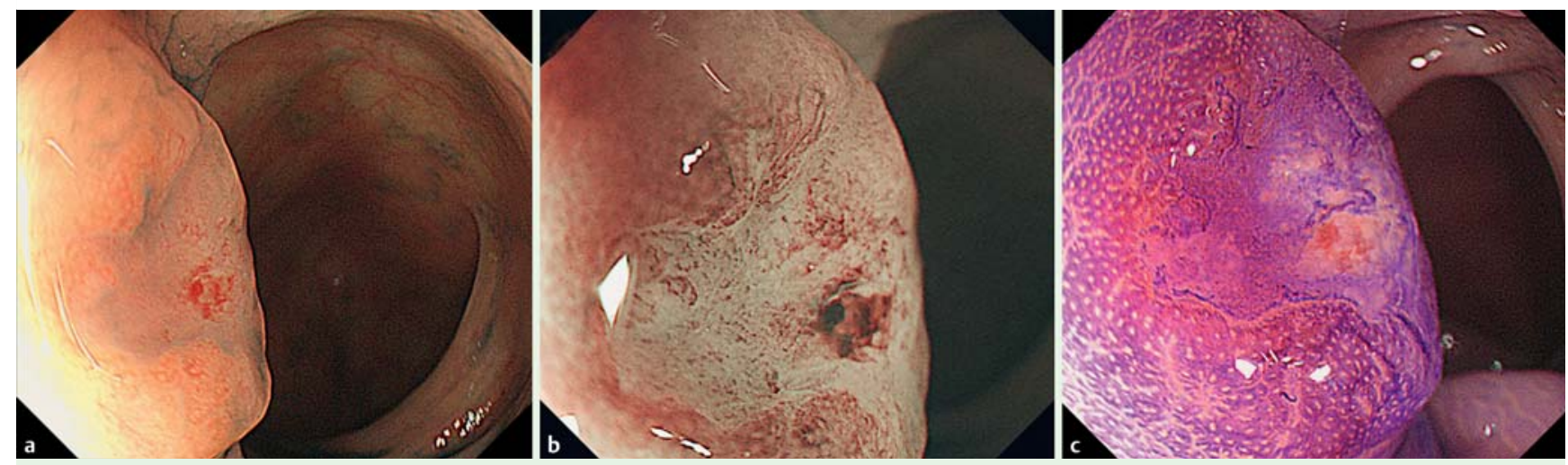

Fig. 2 a Chromoendoscopy image showing the slight depression on the surface of the tumor. b Magnification endoscopy with narrow-band imaging showing the clear distinction between the normal and cancerous mucosa on the surface (demarcated area) and the presence of a nearly avascular or loose microvascular area. These findings represent capillary pattern type IIIB based on the Sano classification. c Magnification endoscopy with Crystal Violet staining showing the decreased number of pits and the amorphous structure of the depressed lesion.

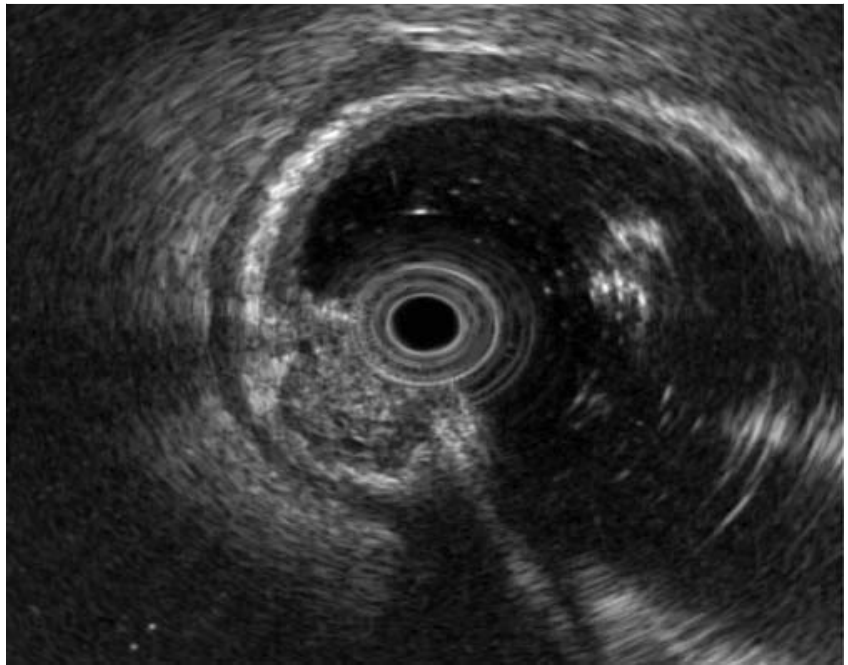

Fig. 3 Endoscopic ultrasonography demonstrating a hypoechoic tumor and isoechoic imaging of the second layer. The tumor appeared relatively hypoechoic at the base and resembled a hypoechoic focus.

endoscopy with Indigo Carmine showed that the margin of the tumor was covered with normal epithelium but that there was a slight depression on the surface of the tumor ( $\bullet$ Fig. 2a). Magnification endoscopy with narrow-band imaging (ME-NBI) revealed that the depressed lesion had a demarcated area and a nearly avascular and loose microvascular area ( $\bullet$ Fig. $\mathbf{2 b}$ ), and it was classified as capillary pattern type IIIB according to the Sano classification [3]. Magnification endoscopy with Crystal Violet staining showed a decrease in the number of pits and showed the amorphous structure of the depressed lesion ( $\bullet$ Fig. 2 c), which was distinguished by a type VN pit pattern [4]; however, the surrounding mucosa showed a normal pit pattern. EUS revealed that the tumor was hypoechoic with a slightly hyperechoic component compared with the second layer and a peripherally hypoechoic rim, resembling a hypoechoic focus ( $\bullet$ Fig. 3). Based on the ME-NBI and EUS findings, we considered a neuroendocrine tumor, malignant lymphoma, or SMT-like adenocarcinoma in the differential diagnosis. A biopsy specimen collected from the surface of the lesion indicated adenocarcinoma. We diagnosed the patient to have adenocarcinoma with massive submucosal invasion of the sigmoid colon and performed a sigmoidectomy. Histologic examination revealed that the lesion was located in the subepithelial layer ( $\bullet$ Fig. 4a), and a well-circumscribed and intense lymphocytic infiltrate surrounded the invasive well-differentiated adenocarcinoma with cystically dilated tumor glands in the submucosal layer ( Fig. $\mathbf{4 b}, \mathbf{c}$ ). The tumor cells were positive for two mismatch repair proteins (MLH1 [ $\bullet$ Fig. 5 a,b] and MSH2 [ $\bullet$ Fig. 5c, d]), suggesting a microsatellite-stable phenotype. The absence of EBV in the carcinoma cells was confirmed by in situ hybridization. We finally diagnosed the tumor as a dome-like carcinoma with lymphoid stroma of the sigmoid colon.

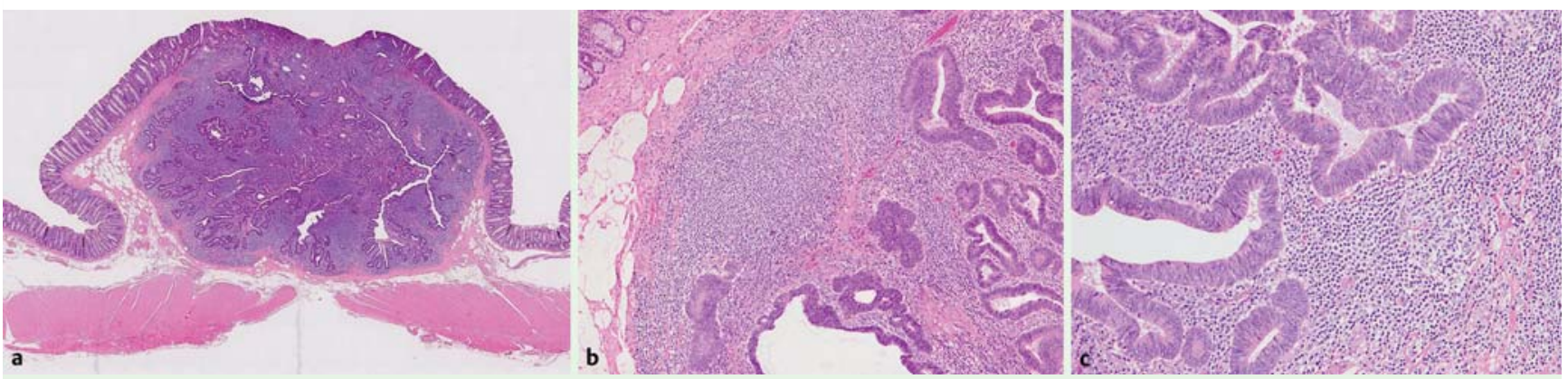

Fig. 4 Histologic appearance of the lesion. Hematoxylin and eosin staining showed the following: a the lesion was located in the subepithelial layer in the panoramic view; $\mathbf{b}$ the infiltrative growth of a well-differentiated adenocarcinoma in the submucosal layer; $\mathbf{c}$ intense lymphocytic infiltrate surrounded the invasive, well-differentiated adenocarcinoma under high-power magnification. Some of the glands were partially cystically dilated. 


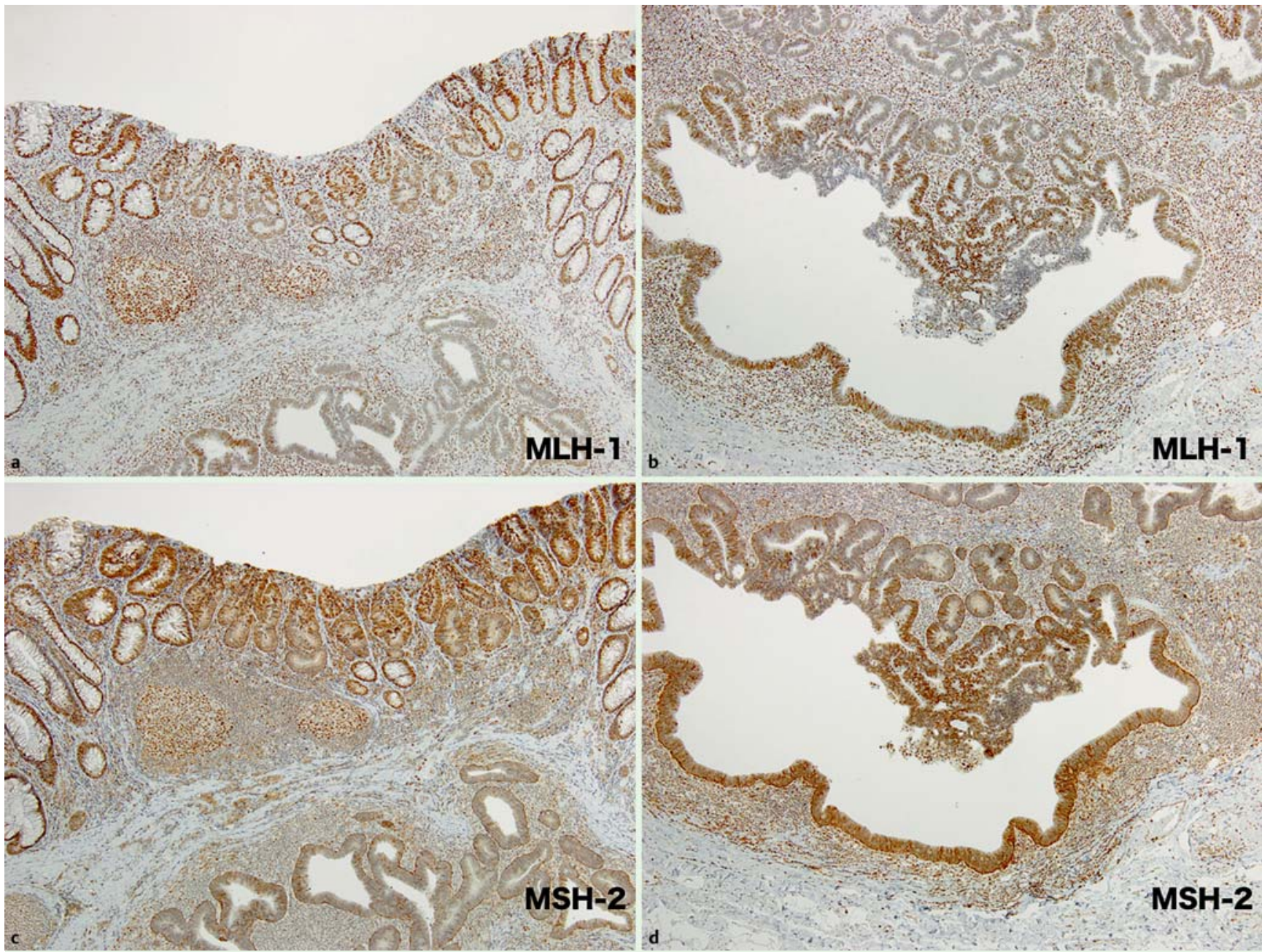

Fig. 5 Immunohistochemical analysis of MLH-1 and MSH-2 protein expression in the tumor. The cancer cells and internal controls (lymphocytes) were positive for MLH-1 (a, b) and MSH-2 (c, d).

\section{Discussion}

$\nabla$

Adenocarcinomas are neoplasms derived from the epithelium. Therefore, an SMT-like appearance is an unusual phenotype of colorectal adenocarcinoma and a rare phenotype in general. It is sometimes difficult to distinguish SMTs from adenocarcinomas that are concealed by normal mucosa and that demonstrate an SMT-like appearance. In this case, white light observation revealed that the lesion resembled an SMT, but chromoendoscopy with Indigo Carmine showed a slight depression on its surface. Moreover, ME-NBI revealed that the depressed lesion had a demarcated area and a nearly avascular and loose microvascular area. These findings indicated adenocarcinoma.

For cases involving a colorectal tumor, such as an SMT, we always consider SMTs, such as neuroendocrine tumors, gastrointestinal stromal tumors, or lymphomas, in the differential diagnosis, which account for most of these types of neoplasm. In such cases, ME-NBI can conveniently distinguish adenocarcinoma from SMT if the epithelial surface of the tumor is exposed and a targeted biopsy can be achieved.

Adenocarcinomas, such as SMTs, are usually pathologically categorized into three types of neoplasm based on their origin as a non-polypoid tumor, mucinous tumor, or tumor with lymphoid stroma. The former two types are predominant, but a tumor with lymphoid stroma or lymphocytic infiltrate is quite rare in the colon.

A carcinoma that is characterized by intense lymphocytic infiltrate is generally termed an LELC. In 1995, Vilor and Tsutsumi first reported a case of LELC in the colon [5]. Generally, LELC is an undifferentiated carcinoma or medullary carcinoma with lymphoid stroma that is typically associated with EBV infection and/ or microsatellite instability-high colorectal cancer. Jass and colleagues subsequently reported a case of dome-like carcinoma of the colon in 2000 with a similar phenotype to that of LELC $[2,6]$. This type of tumor is characterized by a well- and/or moderately differentiated histology with lymphoid stroma and cystically dilated tumor glands [2], in contrast to LELC. Additionally, it is not associated with EBV infection and typically has a microsatellitestable phenotype. The first report by Jass and colleagues led to some confusion as to the differentiation between LELC and dome-like carcinoma [7,8]; however, after it was published, additional cases of dome-like carcinomas were reported $[9,10]$. These tumors have been recognized as dome-like carcinomas, which are distinct from LELC, although both LELC and dome-like carcinomas are relatively rare.

LELC and dome-like carcinoma of the colon also share similar endoscopic findings with developmental features similar to those of SMTs. However, few reports have described EUS findings for both LELC and dome-like carcinoma of the colon. We retrospec- 
tively reviewed the EUS images ( $\bullet$ Fig.3) and found that the panoramic views of the tumors were pathologically similar ( $\bullet$ Fig.4a). The hypoechoic tumor identified by EUS may correspond to the lymphoid stroma, which was composed of carcinoma cells and lymphocytic infiltrates, and may represent a hypoechoic focus-like lesion with the appearance of a dilated gland. Therefore, EUS findings may be helpful in the consideration of dome-like carcinoma when a diagnosis of adenocarcinoma based on a biopsy is rendered. However, it may be difficult to distinguish dome-like carcinoma from LELC by EUS.

Although LELC and dome-like carcinoma are rare and are not distinguishable by the current classification system, they are identified based on morphology (i.e. on the differentiation of the tumor), EBV infection status, and microsatellite instability. Therefore, evaluation of additional cases involving both types of tumors is required to establish SMT-like phenotypes of colorectal adenocarcinoma.

\section{Competing interests: None}

\section{Institutions}

${ }^{1}$ Department of Gastroenterology, Toyonaka Municipal Hospital, Toyonaka, Osaka, Japan

2 Department of Surgery, Toyonaka Municipal Hospital, Toyonaka, Osaka, Japan

${ }^{3}$ Department of Pathology, Toyonaka Municipal Hospital, Toyonaka, Osaka, Japan

\section{References}

1 Carbone A, Micheau C. Pitfalls in microscopic diagnosis of undifferentiated carcinoma of nasopharyngeal type (lymphoepithelioma). Cancer 1982; 50: $1344-1351$

2 Jass JR, Constable L, Sutherland $R$ et al. Adenocarcinoma of colon differentiating as dome epithelium of gut-associated lymphoid tissue. Histopathology 2000; 36: $116-120$

3 Uraoka T, Saito Y, Ikematsu $H$ et al. Sano's capillary pattern classification for narrow-band imaging of early colorectal lesions. Dig Endosc 2011; 23: $112-115$

4 Tanaka S, Kaltenbach T, Chayama K et al. High-magnification colonoscopy (with videos). Gastrointest Endosc 2006; 64: 604-613

5 Vilor M, Tsutsumi Y. Localization of Epstein-Barr virus genome in lymphoid cells in poorly differentiated adenocarcinoma with lymphoid stroma of the colon. Pathol Int 1995; 45: 695-697

6 Clouston $A D$, Clouston DR, Jass JR. Adenocarcinoma of colon differentiating as dome epithelium of gut-associated lymphoid tissue. Histopathology 2000; 37: 567

7 de Petris G, Lev R. Adenocarcinoma of colon associated with gut-associated lymphoid tissue. Histopathology 2000; 37: 566

8 De Petris G, Lev R, Quirk DM et al. Lymphoepithelioma-like carcinoma of the colon in a patient with hereditary nonpolyposis colorectal cancer. Arch Pathol Lab Med 1999; 123: 720-724

9 Asmussen L, Pachler J, Holck S. Colorectal carcinoma with dome-like phenotype: an under-recognised subset of colorectal carcinoma? J Clin Pathol 2008; 61: 482-486

10 Yamada M, Sekine S, Matsuda T et al. Dome-type carcinoma of the colon; a rare variant of adenocarcinoma resembling a submucosal tumor: a case report. BMC Gastroenterol 2012; 12: 21 